\title{
Magnetoresistance in La- and Ca-doped $\mathrm{YBa}_{2} \mathrm{Cu}_{3} \mathrm{O}_{7-\delta}$
}

\author{
B. Qian and J. H. Xing \\ Laboratory of Solid State Microstructures, Department of Physics, Nanjing University, Nanjing 210093, \\ China and Jiangsu Laboratory of Advanced Functional Materials, Department of Physics, \\ Changshu Institute of Technology, Changshu 215500, China
}

\author{
X. S. Wu ${ }^{a)}$ \\ Laboratory of Solid State Microstructures, Department of Physics, Nanjing University, Nanjing 210093, \\ China, Jiangsu Laboratory of Advanced Functional Materials, Department of Physics, Changshu \\ Institute of Technology, Changshu 215500, China, and Department of Physics, The University \\ of Hong Kong, Pokfulam Road, Hong Kong, China \\ S. S. Jiang \\ Laboratory of Solid State Microstructures, Department of Physics, Nanjing University, \\ Nanjing 210093, China \\ J. Gao \\ Department of Physics, The University of Hong Kong, Pokfulam Road, Hong Kong, China
}

(Presented on 2 November 2005; published online 25 April 2006)

We studied the microstructures, electronic, and magnetic properties on La-doped and La- and Ca-codoped $\mathrm{YBa}_{2} \mathrm{Cu}_{3} \mathrm{O}_{7-\delta}(\mathrm{YBCO})$. The superconducting transition temperature remains unchanged up to $10 \%$ for La-doped YBCO. The competition between electrons and holons was assumed according to the variation of $T_{c 0}$ in La and Ca codopings in YBCO. The magnetoresistance (MR) effect is about $8 \%$, which is observed obviously near the critical temperature and is independent of the content of La in La-doped YBCO. MR increases up to about $40 \%$ with the incorporation of $\mathrm{Ca}$ in La-doped YBCO. We present here possible explanations for the magnetoresistance effect in polycrystalline samples based on the microstructure and the increase of oxygen vacancies at grain-boundary interface. (C) 2006 American Institute of Physics.

[DOI: $10.1063 / 1.2173621]$

\section{INTRODUCTION}

Since high temperature superconductors (HTSCs) came into being at the end of $1986,{ }^{1}$ extensive researches have been reported due to large-scale applications of high temperature superconductors. However, polycrystallized materials support significantly lower current densities than single crystals. ${ }^{2-8}$ There is a great deal of interests in improving the superconducting critical current density $\left(J_{c}\right)$ but without affecting other superconducting properties.

It is well known that polycrystalline $\mathrm{YBa}_{2} \mathrm{Cu}_{3} \mathrm{O}_{7-\delta}$ (YBCO) is a weakly coupled granular superconductor., ${ }^{9,10}$ The superconducting critical current density across a grain boundary drops exponentially if the misorientation angle among the grain boundaries exceeds $2^{\circ}-7^{\circ}$. The critical transition width in $R$ - $T$ curve with the existence of HTSC grain boundaries exhibits a large variation with magnetic field applied, which shows the magnetoresistance (MR) effect. ${ }^{11}$ The increase of resistivity near the critical temperature with magnetic field applied is due to the flux-line lattice motion, which can be explained by a phase-slip model. ${ }^{12}$ The model describes the effects of thermal fluctuation of the phase of the order parameters across a highly damped, current driven Josephson junction.

In the present paper we have studied the microstructures, electronic, and magnetic properties on La-doped and La- and

${ }^{a)}$ Electronic mail: xswu@nju.edu.cn
Ca-codoped $\mathrm{YBa}_{2} \mathrm{Cu}_{3} \mathrm{O}_{7-\delta}(\mathrm{YBCO})$. An obvious MR is obtained. By considering the Josephson-like coupling among the $\mathrm{CuO}_{2}$ layers, together with the grain-boundary Josephson junction effects, a possible explanation for the MR in our polycrystalline samples is presented.

\section{EXPERIMENT}

Polycrystalline $\mathrm{YBa}_{2-x} \mathrm{La}_{x} \mathrm{Cu}_{3} \mathrm{O}_{7-\delta}(\mathrm{YBLCO}, x<0.50)$ and $\mathrm{Y}_{0.8} \mathrm{Ca}_{0.2} \mathrm{Ba}_{2-x} \mathrm{La}_{x} \mathrm{Cu}_{3} \mathrm{O}_{7-\delta}$ (YCBLCO, $x<0.50$ ) were prepared using the standard solid-state reaction technique. Details of the sample preparation were described elsewhere. $^{13,14}$

The electric and magnetic properties were characterized by the standard four-probe method. A $4 \mathrm{~T}$ magnetic field was applied to detect the magnetic-field-induced effect on resistivity. Electrical contacts were made with silver glue.

The samples used for structural characterization were ground into powders with the particle size of about $10-30 \mu \mathrm{m}$. The grinding powders were then gently pressed into a standard sample holder and carefully serrated with a razor blade to make a smooth surface and also to minimize any possible preferred orientation in the specimen.

The diffraction data for the Rietveld analysis were collected at room temperature $(\sim 298 \mathrm{~K})$ with Siemens D5000 powder diffractometer in the Bragg-Brentano geometry. The data were collected over the diffraction angle range of $20^{\circ}-$ $100^{\circ}$. No impurity phases were detected in our samples. 


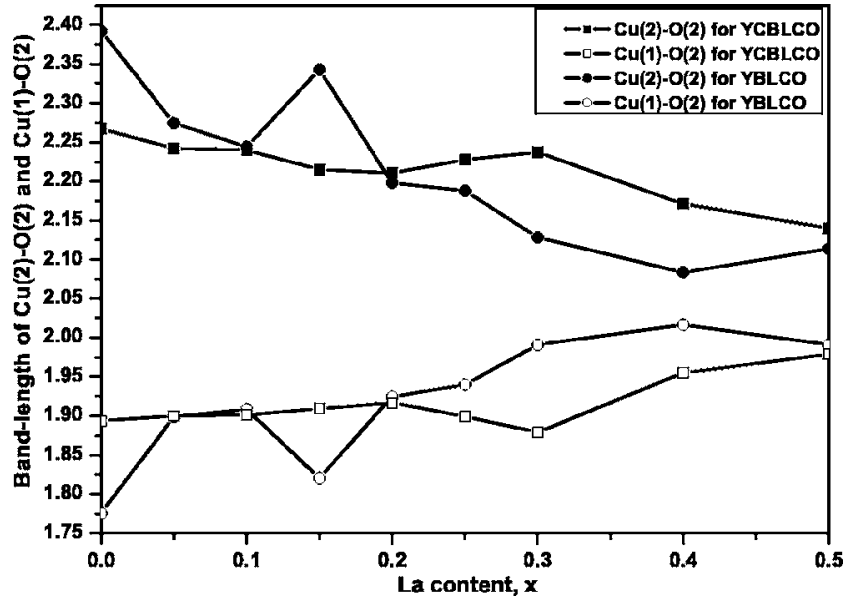

FIG. 1. The bond length $(\AA)$ of $\mathrm{Cu}(2)-\mathrm{O}(2)$ and $\mathrm{Cu}(1)-\mathrm{O}(2)$, determined by Rietveld refinement from x-ray diffraction data vs $x$.

\section{RESULTS AND DISCUSSION}

The x-ray diffraction (XRD) data for all samples can be analyzed in an orthorhombic symmetry with the space group of Pmmm. Rietveld refinements ${ }^{15}$ were used to obtain the atomic parameters in the unit cell. The index of each atom is inconsistent with that reported in Ref. 16. The diffraction background was refined using a fifth order polynomial. A pseudo-Voigt function, which is the linear combination of Gaussian and Lorenz functions, was used to describe the peak profiles. No absorption correction was applied to the raw data. From the fitting, La prefers to occupy the Ba sites and $\mathrm{Ca}$ the $\mathrm{Y}$ site. La can incorporate in YBCO unit cell in a high doping level of $25 \%(x=0.5)$ for La replacing $\mathrm{Ba}$, and $\mathrm{Ca} 50 \%(y=0.5)$ for $\mathrm{Ca}$ substituting for $\mathrm{Y}$, inconsistent with our previous results. ${ }^{13,14}$

The bond length of $\mathrm{Cu}(2)-\mathrm{O}(2)$, which is calculated from the refined atomic parameters, in YCBLCO is larger than that in YBLCO, which is shown in Fig. 1. This indicates that the oxygen atoms sited at $\mathrm{O}(2)$ site shift to the $\mathrm{CuO}$ chains with $\mathrm{Ca}$ incorporation in YBLCO. The apical O(2) in $\mathrm{CuO}_{6}$ octahedral in high- $T_{c}$ cuprates is well known as playing important roles in charge transfer. It is generally believed that superconductivity occurs in the two-dimensional $\mathrm{CuO}_{2}$ sheets. The conduction behavior in the $\mathrm{CuO}_{2}$ planes results from excess holes supplied by the one-dimensional $\mathrm{CuO}$ chains through the oxygen sited at $\mathrm{O}(2)$ sites in the unit cell. The $\mathrm{CuO}$ chains work as the insulating charge reservoirs in the electronic transportation. The carrier concentration in the reservoirs can be governed by the oxygen content in $\mathrm{Cu}-\mathrm{O}$ chains, the balance among the cation ions, and lattice distortions.

$\mathrm{As} \mathrm{La}^{3+}$ has the smaller ionic radius, but high valence than that of $\mathrm{Ba}^{2+}$, La substituting for Ba in YBCO may result in the decrease of the lattice constant along the $c$ direction but induce the increase of electronic carriers in the unit cell. The lattice parameters may be increased with $\mathrm{Ca}$ incorporating in La-doped YBLCO because of the larger ion radius of $\mathrm{Ca}$ ion than that of $\mathrm{Y}$ ion for $\mathrm{Ca}$ substituting for $\mathrm{Y}$ in YBLCO. The composition of La dependences of the lattice parameter of $c$ for YBLCO and YCBLCO is summarized in

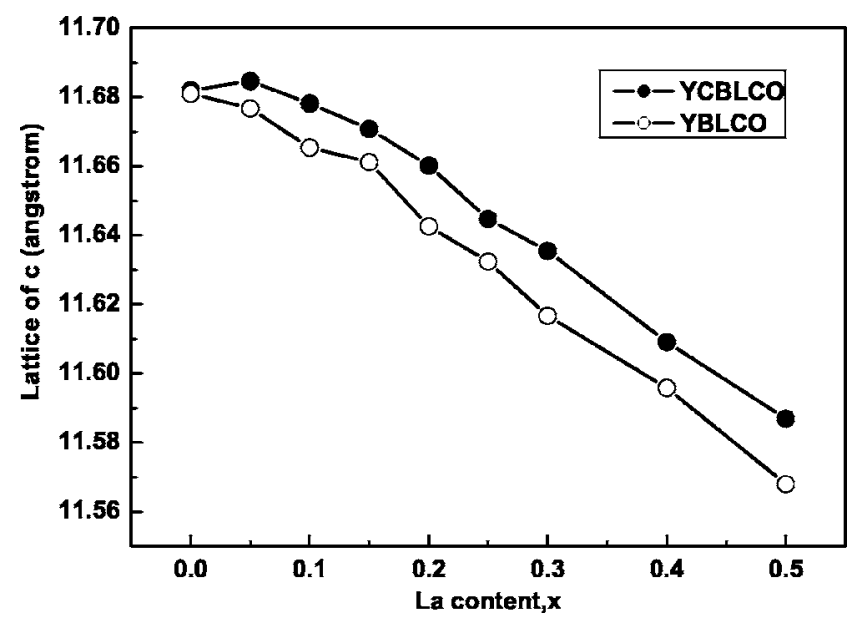

FIG. 2. The La-content dependence of lattice constant of $c(\AA)$ in YBLCO and YCBLCO, determined by Rietveld refinements from x-ray diffraction data vs $x$.

Fig. 2. On the other hand, $\mathrm{Ca}^{2+}$ has the lower valence than that of $\mathrm{Y}^{3+}$, more hole carriers will be introduced when $\mathrm{Ca}$ substitutes for $\mathrm{Y}$ in YCBLCO. The competition between holes and electrons in YCBLCO may occur, which may affect the superconducting behavior in both YBLCO and YCBLCO. We also note that the orthorhombic strain, as described in Refs. 13 and 14, decreases with increasing $\mathrm{La}$ content in both YBLCO and YCBLCO. By the above analysis, we can explain the superconducting behavior in YBLCO and in YCBLCO, just like in Refs. 13 and 14.

Figure 3 shows the typical resistivity $\rho$ as a function of temperature. All $\rho(T)$ curves were measured using a standard four-probe method with or without a magnetic field $H$ applied. The applied magnetic field is perpendicular to the current direction along the normal of the sample surface. The $\rho(T)$ curves without magnetic field applied are consistent with that reported in Ref. 13 and 14. The superconducting critical temperature $T_{c}$, remains unchanged up to $x=0.20$ but decreases with further increasing La content in YBLCO. With Ca corporation into YCBLCO, $T_{c}$ increases with increasing La content up to $x=0.20$ but decreases with further increasing La content. By the superconducting-normal (S-N) model,${ }^{17}$ we can explain the superconducting behavior as the variation of carriers and the distortion in $\mathrm{CuO}_{6}$.

In the experiment for the $R-T$ measurements, we find

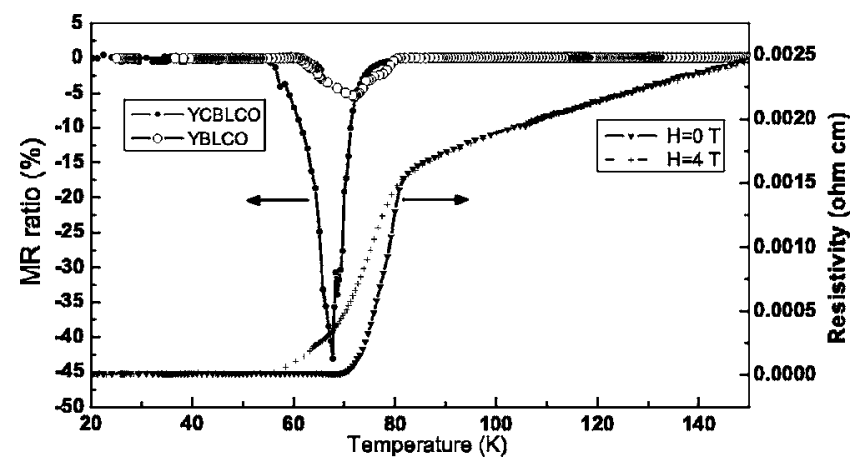

FIG. 3. Typical temperature dependence of resistivity and magnetoresistance as a function of temperature are shown. A $4 \mathrm{~T}$ magnetic field was applied for the determination of the MR effect. 
that $R(T)$ curve of the composite exhibits a strong dependence on the transport current and the magnetic field. The typical variation in resistivity with applied magnetic field is shown in Fig. 3. A magnetoresistance was observed. For convenience, we define here the MR ratio as $\operatorname{MR}(T)=\{[R(0, T)$ $-R(H, T)] / R(0, T)\} 100 \%$, where $R(0, T)$ and $R(H, T)$ are the resistance under the magnetic field of zero and of $H$, respectively, at the temperature of $T$. From the calculation, we find that the maximum MR value (at the valley of MR- $T$ curves, see, for example, in Fig. 3) is about $8 \%$ and remains nearly unchanged, i.e., MR is independent of the content of $\mathrm{La}$ in YBLCO. However, the maximum MR value jumped to about $30 \%-40 \%$ with $\mathrm{Ca}$ incorporation into YBLCO, which is almost five times larger than Ca-free YBLCO.

The effect of the thermally active phase slippage (TAPS) on the dc Josephson effect of single overdamped Josephson junctions has been worked out by Ambegaokar and Halperin. ${ }^{12}$ Because of the short coherence length in high- $T_{c}$ cuprates the superconducting order parameter reduces at interfaces and surfaces, which results in a reduction of the Josephson coupling through the high- $T_{c}$ grain-boundary Josephson junctions, and TAPS is then expected to be present over a wide temperature range below the critical temperature.

Based on the above theory, when $k_{B} T$ becomes in comparable with the Josephson coupling energy, the resistivity $\rho(T)$ in the limit of low current is given by $\rho(T)$ $=\rho_{n}\left[I_{0}(\gamma / 2)\right]^{-2}$, where, $I_{0}$ is the modified Bessel function, $\rho_{n}$ is the average normal state resistivity of the junction, and $\gamma$ is the normalized barrier height for thermally active phase slip, defined as $\gamma=U_{0} / k_{B} T=A(1-t)^{q} / H$. Here, $U_{0}$ is the active energy, $t \equiv T / T_{C}$ is the reduced temperature, $H$ is the applied magnetic field, and $A$ is a constant. With the increase of magnetic field, the coupling energy between the Josephson vortices decreases. The value of $\gamma$ also decreases with cation doping in YBCO. Meanwhile the homogeneity of the sample varies with the increase of the dopants, which may work as the weak links. The weak link resulting from both the doping and the grain boundaries works like Josephson junction and induces the MR effect.

Many research groups ${ }^{18,19}$ suggested that there are significant Josephson couplings among $\mathrm{CuO}_{2}$ planes because the coherence length along the $c$ axis is greater than the spacing distance between the $\mathrm{CuO}_{2}$ planes. The spacing distance between two $\mathrm{CuO}_{2}$ planes remains almost the same for La doping in YBLCO but varies with $\mathrm{Ca}$ incorporation into YBLCO, as obtained from the Rietveld refinements above. The above results indicate that the coupling energy of the Josephson junctions between $\mathrm{CuO}_{2}$ planes is almost unchanged for YBLCO but decreases with $\mathrm{Ca}$ incorporation into YBLCO, which induced the obvious increase in resistivity under magnetic field.
The orthorhombic strain, which is defined as $\sigma$ $=[2(a-b) /(a+b)] 100 \%$, decreases with increasing La content and also with $\mathrm{Ca}$ incorporation into YBLCO. According to Jorgensen et al., ${ }^{20}$ and our previous works, ${ }^{13,14,21,22}$ the decrease in orthorhombic strain and in lattice parameter of $c$ indicate the increase of oxygen vacancy in $\mathrm{CuO}$ chain in YBCO. Klie et al. ${ }^{11}$ pointed out that in bulk YBCO, the formation energy of oxygen in $\mathrm{CuO}_{2}$ sheet $(\sim 3.3 \mathrm{eV})$ at the boundary is about $0.7 \mathrm{eV}$ higher than that in the $\mathrm{CuO}$ chains $(\sim 2.6 \mathrm{eV})$ at the boundary. Therefore, the grain boundaries in YBCO contain more oxygen vacancies, which results in the decrease of the grain-boundary charge and the aggregation of the space charge. The carrier transport is then suppressed, which destroys the $e-e$ pairs and also the superconducting transition $T_{c}$ decreases. The oxygen vacancy and pin flux with magnetic field applied at the grain boundaries may increase; the pinning flux may scatter the transport band electrons and increase the resistivity. A positive MR effect then can be observed.

\section{ACKNOWLEDGMENTS}

This work has been supported by NNSFC (Grant Nos. 10474031, 10523001, and 90201039). The authors would like to thank the State Key Project of Fundamental Research (Grant No. 001CB610602) and the special project from the Department of Chinese Education (No. 20020284029).

${ }^{1}$ J. G. Bednorz and K. A. Müller, Z. Phys. B: Condens. Matter 64, 189 (1986).

${ }^{2}$ D. Dimos, P. Chaudhari, and J. Mannhart, Phys. Rev. B 41, 4038 (1990). ${ }^{3}$ R. Gross and B. Mayer, Physica C 180, 235 (1991).

${ }^{4}$ A. Gurevich and E. A. Pashitskii, Phys. Rev. B 57, 13878 (1998).

${ }^{5}$ N. F. Heinig, R. D. Redwing, I. F. Tsu, A. Gurevich, J. E. Nordman, S. E. Babcock, and D. C. Larbalestier, Appl. Phys. Lett. 69, 577 (1996).

${ }^{6} \mathrm{H}$. Hilgenkamp and J. Mannhart, Rev. Mod. Phys. 74, 485 (2002).

${ }^{7}$ J. Mannhart, P. Chaudhari, D. Dimos, C. C. Tsuei, and T. R. McGuire, Phys. Rev. Lett. 61, 2476 (1988).

${ }^{8}$ P. W. Anderson and Z. Zou, Phys. Rev. Lett. 60, 132 (1988).

${ }^{9}$ K. A. Müller, M. Takashige, and J. G. Bednorz, Phys. Rev. Lett. 58, 1143 (1987).

${ }^{10}$ D. K. Finnemore et al., Phys. Rev. B 35, 5319 (1987).

${ }^{11}$ R. F. Klie, J. P. Buban, M. Varela, A. Franceschetti, C. Jooss, Y. Zhu, N. D. Browning, S. T. Pantelides, and S. J. Pennycook, Nature (London) 435, 475 (2005)

${ }^{12}$ H. Ambegaokar and B. I. Halperin, Phys. Rev. Lett. 22, 1364 (1969).

${ }^{13}$ X. S. Wu and J. Gao, Physica C 315, 215 (1999).

${ }^{14}$ X. S. Wu, F. Z. Wang, J. S. Liu, S. S. Jiang, and J. Gao, Physica C 320, 206 (1999).

${ }^{15}$ R. A. Young, J. Appl. Crystallogr. 28, 366 (1995).

${ }^{16}$ X. S. Wu, S. S. Jiang, C. C. Lam, D. W. Wang, X. R. Huang, Z. H. Wu, Y. Xuan, and X. Jin, Phys. Status Solidi A 157, 439 (1996).

${ }^{17}$ D. N. Shi and Z. Z. Li, Physica C 270, 274 (1996).

${ }^{18}$ G. Deutscher and K. A. Müller, Phys. Rev. Lett. 59, 1745 (1987).

${ }^{19}$ R. L. Peterson and J. W. Ekin, Phys. Rev. B 37, 9848 (1988).

${ }^{20}$ J. D. Jorgensen, B. W. Veal, A. P. Paulikis, L. J. Lowicki, and G. W. Grabtree, Phys. Rev. B 41, 1863 (1990).

${ }^{21}$ X. S. Wu, S. S. Jiang, J. Lin, J. S. Lin, W. M. Chen, and X. Jin, Physica C 309, 25 (1998).

${ }^{22}$ X. S. Wu and J. Gao, Physica C 313, 79 (1999). 